\title{
Stationary core-annular flow through a horizontal pipe
}

\author{
G. Ooms and P. Poesio \\ J.M. Burgers Center, Delft University of Technology, Laboratory for Aero- and Hydrodynamics, Leeghwaterstraat 21, \\ 2628 CA Delft, The Netherlands
}

(Received 4 August 2003; published 15 December 2003)

\begin{abstract}
A theoretical investigation has been made of core-annular flow: the flow of a high-viscosity liquid core surrounded by a low-viscosity annular liquid layer through a horizontal pipe. Special attention is paid to the question how the buoyancy force on the core, caused by a possible density difference between the core and the annular layer, is counterbalanced. From earlier studies it is known that at the core surface ripples are present that have the shape of "bamboo" waves or "snake" waves. They generate pressure variations and secondary flows in the annular layer that can cause a net hydrodynamic force on the core. Using hydrodynamiclubrication theory (assuming the core to be rigid) it has been shown that for snake waves the lubrication force can counterbalance the buoyancy force. For bamboo waves that is not the case.
\end{abstract}

DOI: 10.1103/PhysRevE.68.066301

PACS number(s): 47.15.-x

\section{INTRODUCTION}

In transporting a high-viscosity liquid through a pipe a low-viscosity liquid can be used as a lubricant film between the pipe wall and the high-viscosity core. This technique, called core-annular flow, is very interesting from a practical and scientific point of view. In a number of cases it was successfully applied for pipeline transport of very viscous oil. The low-viscosity liquid in these cases was water. The pressure drop over the pipeline was considerably lower for oil-water core-annular flow than the pressure drop for the flow of oil alone at the same mean oil velocity.

Much attention has been paid in the literature to coreannular flow. Joseph and Renardy [1] have written a book about it. There are several review articles, see for instance, Oliemans and Ooms [2], and Joseph et al. [3]. Most papers deal with the development of waves at the interface between the high-viscosity liquid and the low-viscosity one, see Ooms [4], Bai et al. [5], Bai et al. [6], Renardy [7], Li and Renardy [8], Kouris and Tsamopoulos [9], and Ko et al. [10]. These studies deal with axisymmetric vertical core-annular flow (the core has a concentric position in the pipe). In that case the buoyancy force on the core, due to a possible density difference between the two liquids, is in the axial direction of the pipe. It was shown experimentally and theoretically that both liquid phases can retain their integrity, although an originally smooth interface was found to be unstable. For vertical upward flows axisymmetric traveling waves develop with slightly sharper crests (pointing toward the annular fluid) than troughs, the so-called "bamboo waves." For vertical downward flows also "corkscrew waves" and "snake waves" are possible, in which case the interface is not only dependent on the axial direction of the pipeline but also on the tangential direction (although the core remains concentric in the pipe). Using the calculated wave form also predictions were made for the pressure drop over the pipe and the hold-up's of the liquids.

For the transport of very viscous oil (or other liquids) it is also important to pay attention to core-annular flow through a horizontal pipe. Since the densities of the two liquids are almost always different, gravity will push the core off-center in that case. Experimental results suggest that under normal conditions a steady eccentric core-annular flow (rather than a stratified flow) is achieved. It can be shown that for a steady flow a wavy interface is needed to levitate the core. Relatively little attention has been given to the explanation of this levitation mechanism. Ooms [4], Ooms and Beckers [11], Ooms et al. [12], and Oliemans and Ooms [2] proposed a mechanism based on hydrodynamic lubrication theory. They showed that levitation could not take place without a hydrodynamic lifting action due to the waves present at the oilwater interface. In their work they assumed that the core viscosity is infinitely large. So any deformation of the interface was neglected and the core moved as a rigid body at a certain speed with respect to the pipe wall. The shape of the waves was given as empirical input. They were assumed to be sawtooth waves that were like an array of slipper bearings and pushed off the core from the wall by lubrication forces. In their case the core would be sucked to the pipe wall if the velocity was reversed. So the slipper bearing picture is obligatory if levitation is wanted. However it was pointed out by Bai et al. [5] that (at finite oil viscosity) the sawtooth waves are unstable since the pressure is highest just where the gap between the core and the pipe wall is smallest. So the wave must steepen where it is gentle and become smooth where it is sharp, and levitation of the core due to lubrication forces is no longer possible. To get a levitation force from this kind of wave inertial forces are needed according to Bai et al. [5].

From their study of the wave development for a concentric vertical core-annular flow Bai et al. [5] tried to draw some conclusions about the levitation force on the core in case of an eccentric horizontal core-annular flow. They considered what might happen if the core moved to a slightly eccentric position owing to a small difference in density. The pressure distribution in the liquid in the narrow part of the annulus would intensify and the pressure in the wide part of the annulus would relax according to their predicted variation of pressure with the distance between the core and the pipe wall for the concentric case. In that case a more positive pressure would be generated in the narrow part of the annulus which would levitate the core. It is important to point out 


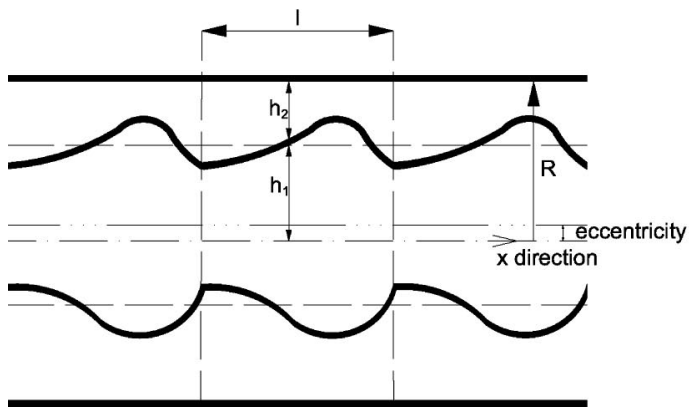

again that the study of Bai et al. [5] was for a concentric core. In a horizontal core-annular flow with a density difference between the liquids the core will be in an eccentric position and due to the presence of waves at the interface secondary flows perpendicular to the pipe axis are generated. This type of secondary flows that also contribute to the force on the core is not considered in concentric core-annular flow.

Another core levitation model was proposed by Bannwart [13]. It is based on an interface-curvature-gradient effect associated with interfacial tension: if the radius of curvature increases with $\theta$ (the tangential coordinate, see Fig. 1) a downward force acts on the core due to interfacial tension. In this paper we will not make a comparison of the relative importance of the three levitation mechanisms, levitation due to (1) hydrodynamic-lubrication forces, (2) pressure forces caused by inertia, and (3) interfacial-tension forces. Here we concentrate on a further development of the hydrodynamiclubrication model.

The fact that a levitation of the core does not come from lubrication forces but from inertial forces was proved by Bai et al. [5] for cylindrically symmetric waves. The waves that were used in their calculation were bamboo waves that were periodic in the axial direction of the pipe and were independent of the tangential coordinate. As found by Renardy [7] waves are possible that are not cylindrically symmetric; waves that are dependent on the $x$ direction and also on the tangential direction (for instance, snake waves). It seems evident that for such waves the forces on the core and also the secondary flow in the annulus will be different than for a core with bamboo waves. So it is interesting to study the levitation forces on the core for the case of noncylindrically symmetric waves and to investigate the possible contribution of hydrodynamic lubrication to these forces. In this paper we will show that core levitation by lubrication forces alone is possible for snake waves, contrary to the result for bamboo waves. As in the earlier work of Ooms and Oliemans [2] we assume that the core viscosity is infinitely large. So the core (with snake waves) moves as a rigid body at a certain velocity with respect to the pipe wall. In our calculations the problem of a moving core is transformed into one in which the core is supposed to be at rest with respect to the reference system and where the pipe wall has a velocity in the axial direction. The starting point of the calculation is the Reynolds equation, the basic equation of the hydrodynamiclubrication theory for the pressure distribution in the annulus. We will solve this equation by means of a perturbation calculation with the dimensionless wave amplitude as perturbation parameter. From the calculated pressure the velocity dis- tribution in the annulus will be derived and the pressure forces and viscous forces exerted on the core are determined. It is then investigated whether a steady situation is possible. For a steady situation to arise a balance is required between the buoyancy force on the core and the vertical components of the pressure forces and viscous forces on the core. We will show that for snake waves such a balance is possible.

\section{THEORY}

As mentioned in the Introduction we start from the Reynolds equation for the liquid flow in the annulus

$$
\frac{\partial}{\partial \theta}\left(\frac{h_{2}^{3}}{R^{2}} \frac{\partial \phi}{\partial \theta}\right)+\frac{\partial}{\partial x}\left(h_{2}^{3} \frac{\partial \phi}{\partial x}\right)=6 \mu W \frac{\partial h_{2}}{\partial x},
$$

where $r, \theta$, and $x$ are the cylindrical coordinates belonging to the pipe. $\theta$ has the value 0 at the top of the horizontal pipe. A frame of reference is chosen, according to which the core is at rest and the pipe wall has a velocity $W$ in the $x$ direction. $h_{2}(\theta, x)$ represents the thickness of the annular space between the core and the pipe wall. $R$ is the radius of the pipe and $\mu$ is the dynamic viscosity of the liquid. In Fig. 1 a sketch of the geometrical configuration is given. (It is pointed out that in this figure the thickness of the annular layer is much too large to be practical for applications. However, the figure is only meant for introducing the relevant geometrical parameters.) The variable $\phi$ is given by

$$
\phi=p+\rho g r \cos \theta,
$$

where $p$ is the hydrodynamic pressure in the liquid in the annulus, $\rho$ is the density of the liquid, and $g$ is the acceleration due to gravity.

The thickness of the annulus can be derived from the relation

$$
h_{1}+h_{2}=R \text {, }
$$

where $h_{1}$ describes the core surface with respect to the pipe axis. We assume the general shape of the core to be given by

$$
\begin{aligned}
h_{1}(\theta, x)= & h_{1}^{(0)}\left[1+\epsilon\left(\sum_{m, n=0}^{\infty} a_{m, n} \cos m \theta \cos \frac{n \pi x}{l}\right.\right. \\
& \left.\left.+\sum_{m, n=0}^{\infty} b_{m, n} \cos m \theta \sin \frac{n \pi x}{l}\right)\right]
\end{aligned}
$$


where $h_{1}^{(0)}$ is the constant radius of the core for the case that no wave is present and $l$ is the wavelength of the wave at the core surface. $\epsilon h_{1}^{(0)} a_{m, n}$ and $\epsilon h_{1}^{(0)} b_{m, n}$ are the Fourier amplitudes of the wave with respect to the core centerline. $\epsilon$ is a constant and assumed to be much smaller than unity. (In the paper of Ooms and Beckers [11] only the terms $a_{0, n}$, $a_{m, 0}, b_{0, n}$, and $b_{m, 0}$ were multiplied by $\epsilon$ in the expression for $h_{1}$. All other terms were multiplied by $\epsilon^{2}$ and were for that reason an order of magnitude smaller. Kronig [14] already criticized this assumption. In this respect the calculation given in this paper in which all terms are of the order $\epsilon$, is an improvement with respect to the earlier calculation.) We will first solve the flow problem for the general shape of Eq. (4) and thereafter we will study the special case that the wave has the form of a snake wave. In Eq. (4) we have omitted terms with $\sin m \theta$, as for symmetry reasons they cannot contribute to a net vertical hydrodynamic force on the core and hence to a compensation of the buoyancy force.

The Reynolds equation is solved by means of a perturbation calculation with $\epsilon$ as the perturbation parameter. So we assume that $\phi$ can be written as

$$
\phi=\sum_{i=0}^{\infty} \epsilon^{i} \phi^{(i)}
$$

After some straightforward calculations we find the following solution in zeroth-order approximation

$$
\phi^{(0)}=\beta x
$$

and in first-order approximation

$$
\begin{aligned}
\phi^{(1)}= & 3 \beta x \sum_{m, n=0}^{\infty} \frac{m}{m^{2}+\left(\frac{n \pi R}{l}\right)^{2}} \\
& \times\left(a_{m, n} \sin m \theta \cos \frac{n \pi x}{l}-b_{m, n} \sin m \theta \sin \frac{n \pi x}{l}\right) \\
& +\frac{(12 \mu W / R)}{\left(\frac{h_{2}^{(0)}}{R}\right)^{4}} \sum_{m, n=0}^{\infty} \frac{\left(\frac{n \pi R}{l}\right)}{m^{2}+\left(\frac{n \pi R}{l}\right)^{2}} \\
& \times\left(a_{m, n} \cos m \theta \cos \frac{n \pi x}{l}-b_{m, n} \cos m \theta \sin \frac{n \pi x}{l}\right),
\end{aligned}
$$

where $\beta$ represents a constant pressure gradient.

In order to be able to calculate the net hydrodynamic force on the core (with contributions from pressure forces and viscous friction forces) we need to calculate also the velocity distribution in the liquid in the annulus. Assuming the thickness of the annulus to be small compared to the pipe radius and the wavelength (the basic assumption of the hydrodynamic-lubrication theory) the equations of motion can be simplified to

$$
\begin{gathered}
\frac{\partial \phi}{\partial r}=0 \\
\frac{1}{r} \frac{\partial \phi}{\partial \theta}=\mu \frac{\partial}{\partial r}\left[\frac{1}{r} \frac{\partial}{\partial r}(v r)\right] \\
\frac{\partial \phi}{\partial x}=\frac{\mu}{r} \frac{\partial}{\partial r}\left(r \frac{\partial w}{\partial r}\right),
\end{gathered}
$$

where $v$ and $w$ represent the velocity components of the liquid in the annulus in the $\theta$ and $x$ directions, respectively. The boundary conditions are

$$
\text { for } r=h_{1}: v=0 \text { and } w=0
$$

and

$$
\text { for } r=R: v=0 \text { and } w=W \text {. }
$$

Integration of the equations of motion (using the boundary conditions) yields

$$
\begin{aligned}
& v=\frac{1}{2 \mu} \frac{\partial \phi}{\partial \theta}\left[r \ln r+\frac{h_{1}^{2}\left(r^{2}-R^{2}\right) \ln h_{1}}{r\left(R^{2}-h_{1}^{2}\right)}-\frac{R^{2}\left(r^{2}-h_{1}^{2}\right) \ln R}{r\left(R^{2}-h_{1}^{2}\right)}\right], \\
& w=\frac{1}{4 \mu} \frac{\partial \phi}{\partial x}\left[r^{2}-R^{2}+\frac{\left(R^{2}-h_{1}^{2}\right) \ln r / R}{\ln h_{1} / R}\right]-\frac{W \ln r / R}{\ln h_{1} / R}+W .
\end{aligned}
$$

As the pressure distribution and velocity distribution in the liquid annulus are now known, we are able to calculate the net vertical pressure-and viscous force exerted on the core. We assume that these forces counterbalance the buoyancy force on the core caused by the difference in density between the core and the liquid in the annulus. It has been shown by Ooms and Beckers [11] that this results in the following condition:

$$
\begin{aligned}
& R \int_{0}^{2 \pi} d \theta \int_{0}^{l} d x(\phi)_{r=R} \cos \theta \\
& \quad+\mu R \int_{0}^{2 \pi} d \theta \int_{0}^{l} d x\left[\frac{\partial}{\partial r}\left(\frac{v}{r}\right)\right]_{r=R} \sin \theta \\
& \quad=\frac{\Delta \rho g}{2} \int_{0}^{2 \pi} d \theta \int_{0}^{l} d x h_{1}^{2} .
\end{aligned}
$$

The first term on the left-hand side represents the contribution due to the pressure forces on the core, the second term represents the contribution due to the viscous forces and the term on the right-hand side represents the buoyancy force. Substitution of the calculated pressure distribution and velocity distribution gives the following condition for stationary core-annular flow (counterbalancing of the buoyancy force by the pressure forces and viscous forces) 


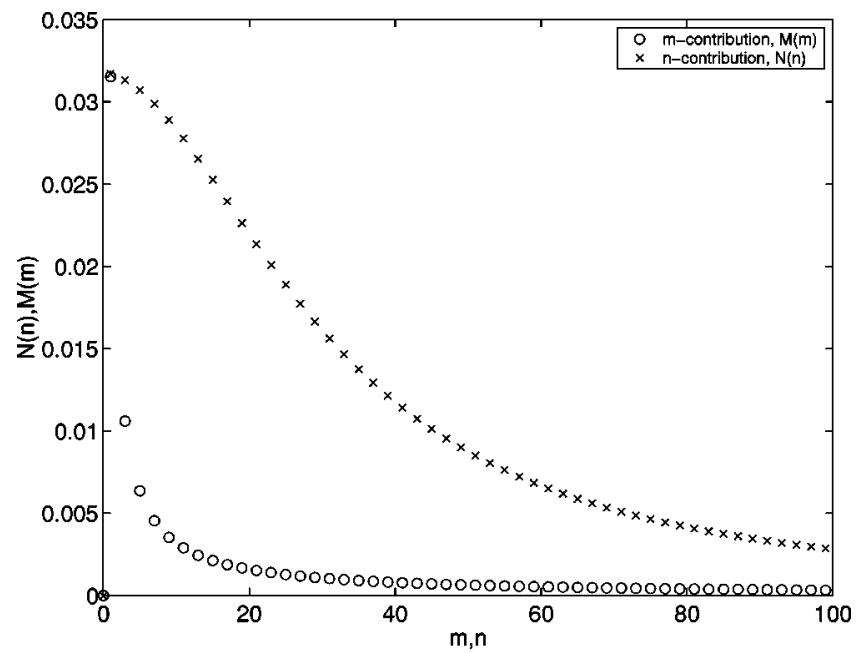

FIG. 2. Contribution of each term in $m$ and $n$. Harmonics $n$ $<100$ for $N(n)$ and $m<20$ for $M(m)$ are the only modes that are relevant.

$$
\begin{gathered}
6 \pi^{2} \mu W \epsilon^{2} l \frac{\left[-2 \ln \left(h_{2}^{(0)} / R\right)^{2}\right]}{\left(h_{2}^{(0)} / R\right)^{4}} \sum_{m, n=0}^{\infty} \frac{\left(\frac{n \pi R}{l}\right)}{(m+1)^{2}+\left(\frac{n \pi R}{l}\right)^{2}} \\
\times\left(a_{m, n} b_{m+1, n}-b_{m, n} a_{m+1, n}\right)=\pi \Delta \rho g l h_{1}^{(0) 2}
\end{gathered}
$$

Equation (16) is very interesting and we make the following remarks about it.

(1) In our calculation we found that the net vertical force on the core is only due to the viscous forces, not due to the pressure forces. The mathematical reason is that the viscous term in Eq. (15) (with the velocity derivative) consists of terms containing a product of $\sin m \theta$ and $\cos (m+1) \theta$ (because of the product of $\partial \phi / \partial \theta$ and the function of $h_{1}$ in the viscous term). Such a product can be written as a sum of two trigonometric functions, one of which is $\sin \theta$. Substitution in Eq. (15) gives a $\sin ^{2} \theta$ term, which after integration results in a net contribution to the vertical hydrodynamic force on the core. This explanation cannot be given for the pressure term in Eq. (15).

(2) Because of the buoyancy force the core will have an eccentric position in the pipe. The eccentricity is given by the term $\epsilon h_{1}^{(0)} a_{1,0} \cos \theta$ in Eq. (4) for the description of the core surface with respect to the pipe centerline. (It can be shown that in first-order approximation with respect to $\epsilon$ this term describes the vertical displacement of a cylinder with respect to its origin.) All other terms $a_{m, n}$ and $b_{m, n}$ describe the wave shape at the core surface.

(3) The denominator of Eq. (16) contains terms with $m^{2}$ and $n^{2}$. As the coefficients $a_{m, n}$ and $b_{m, n}$ are of the order of unity, it follows that the contribution to the vertical hydrodynamic force on the core decreases with increasing order of $m$ and $n$. To illustrate this point we show in Fig. 2 the term $M(m)=\sum_{n=0}^{\infty}\left[(n \pi R / l) /(m+1)^{2}+(n \pi R / l)^{2}\right]$ as function of $m$ and $N(n)=\sum_{m=0}^{\infty}\left[(n \pi R / l) /(m+1)^{2}+(n \pi R / l)^{2}\right]$ as function of $n$. So, for each selected value of $m$ we have included the contributions of all $n$ values. And for each selected value of $n$ we have included the contributions of all $m$ values. As can be seen the lowest values of $m$ and $n$ are most important for the vertical hydrodynamic force.

(4) From Eq. (16) it is clear that no counterbalancing of the buoyancy force is possible for $n=0$. The physical explanation is, of course, that for $n=0$ no wave is present at the core surface. So no core levitation is possible without a wave at the core surface moving with respect to the pipe wall.

(5) If we select $a_{0,1} \neq 0 \quad\left(a_{1,0} \neq 0\right.$ because of core eccentricity) and all other possible combinations of $a_{m, n}=0$ and $b_{m, n}=0$, the wave has the symmetrical shape of a cosine function in the $x$ direction independent of the $\theta$ value. From Eq. (16) it follows that core levitation is not possible for such a wave shape. Stationary core-annular flow is, therefore, only possible when the wave has a nonsymmetrical shape in the $x$ direction or when the wave shape is dependent on $\theta$.

(6) $\beta$ is not present in Eq. (16). So the constant pressure gradient over the pipe has no influence on the levitation force on the core.

Equation (16) can be written in the following dimensionless way:

$$
\frac{\Delta \rho}{\rho}=\frac{F r}{\operatorname{Re}} \epsilon^{2} f\left(H_{2}^{(0)}, L\right),
$$

with the Froude number $\mathrm{Fr}=W^{2} / g R$ and the Reynolds number $\operatorname{Re}=(\rho W R / \mu) . f\left(H_{2}^{(0)}, L\right)$ is a known function of the dimensionless thickness of the annular layer $H_{2}^{(0)}=h_{2}^{(0)} / R$ and the dimensionless wavelength $L=l / R . \rho$ is the density of the liquid. With Eq. (17) we can calculate which dimensionless density difference can be counterbalanced by the lubrication forces as function of the relevant dimensionless groups. In the following section (when we discuss a core with a ripple in the form of a snake wave) we will give some quantitative results derived from this equation.

Ooms and Beckers [11] also proved that the force exerted on the core in the length direction of the pipe per unit of length is given by

$$
F_{x}=\frac{\mu R}{l} \int_{0}^{2 \pi} d \theta \int_{0}^{l} d x\left(\frac{\partial w}{\partial r}\right)_{r=R},
$$

or after substitution of Eq. (14) by

$$
\begin{aligned}
F_{x}= & -\frac{\mu R}{l} \int_{0}^{2 \pi} d \theta \int_{0}^{l} d x\left(\frac{1}{\ln \left(h_{1} / R\right)}\right)+\frac{R^{2}}{4 l} \int_{0}^{2 \pi} d \theta \int_{0}^{l} d x \\
& \times\left(2+\frac{1-\left(h_{1} / R\right)^{2}}{\ln \left(h_{1} / R\right)}\right) \frac{\partial \phi}{\partial x} .
\end{aligned}
$$

The first part of the right-hand side of Eq. (19) represents the contribution due to the main flow in the axial direction of the pipe. When there is no additional pressure gradient in the annular layer $(\beta=0)$, the second part is solely due to the secondary flows perpendicular to the pipe axis (caused by the ripple in the core surface), which are necessary for counterbalancing the buoyancy force. Again in the following section we will give quantitative results derived from this equation for the case of a core with a snake wave. 


\section{BAMBOO WAVES, SAWTOOTH WAVES, AND SNAKE WAVES}

Bamboo waves and sawtooth waves have the same general wave form

$$
\begin{aligned}
h_{1}= & \epsilon h_{1}^{(0)} a_{1,0} \cos \theta+h_{1}^{(0)} \\
& +\left[\epsilon h_{1}^{(0)}\left(\sum_{n=1}^{\infty} a_{0, n} \cos \frac{n \pi x}{l}+\sum_{n=1}^{\infty} b_{0, n} \sin \frac{n \pi x}{l}\right)\right] .
\end{aligned}
$$

As mentioned earlier the first term on the right-hand side represents the eccentricity of the core with respect to the pipe axis, the second term is the constant radius of the core in case of absence of a wave, and the third term represents the wave shape. As can be seen the wave shape is independent of the tangential direction $\theta$. The only difference between a bamboo wave and a sawtooth wave is the shape of the wave in the $x$ direction. It is clear from Eq. (16) that for both types of waves a vertical hydrodynamic force is not found in $\epsilon^{2}$ approximation. However, we know from the work of Ooms and Beckers [11] that when we extend the calculation to higher orders in $\epsilon$ a net vertical force will be found. They found that the sawtooth wave can levitate the core by a net viscous force. The problem is, as discussed in the Introduction, that the sawtooth wave is not hydrodynamically stable and will deform (in case a finite core viscosity is taken into account). Also for a bamboo wave a net viscous force exerted on the core is found in higher orders of $\epsilon$. However, this force is in the same direction as the buoyancy force and the core will therefore be sucked against the pipe wall. As shown by Bai et al. [5] inertial forces are needed for a possible levitation of a core with a bamboo wave.

Snake waves differ from bamboo waves because of their $\theta$ dependence. There are many snake waves (with different $\theta$ dependence) possible. From Eq. (16) we know, however, that for a net levitation force it is necessary that there are at least two Fourier components with successive $m$ values: $m$ and $m+1$. We have selected $m=0$ and $m=1$. Therefore only $a_{0, n}, a_{1, n}, b_{0, n}$ and $b_{1, n}$ are different from zero. All other Fourier components are assumed to be absent. So we choose the following shape for the ripple in the core surface:

$$
\begin{aligned}
h_{1}= & \epsilon h_{1}^{(0)} a_{1,0} \cos \theta+h_{1}^{(0)} \\
& +\left[\epsilon h _ { 1 } ^ { ( 0 ) } \left(\sum_{n=1}^{\infty} a_{0, n} \cos \frac{n \pi x}{l}\right.\right. \\
& \left.\left.+\left(a_{1, n}-a_{1,0}\right) \cos \theta \cos \frac{n \pi x}{l}\right)\right] \\
& +\left[\left(\epsilon h_{1}^{(0)} \sum_{n=1}^{\infty} b_{0, n} \sin \frac{n \pi x}{l}+b_{1, n} \cos \theta \sin \frac{n \pi x}{l}\right)\right] .
\end{aligned}
$$

Again the first term on the right-hand side represents the eccentricity of the core with respect to the pipe axis, the

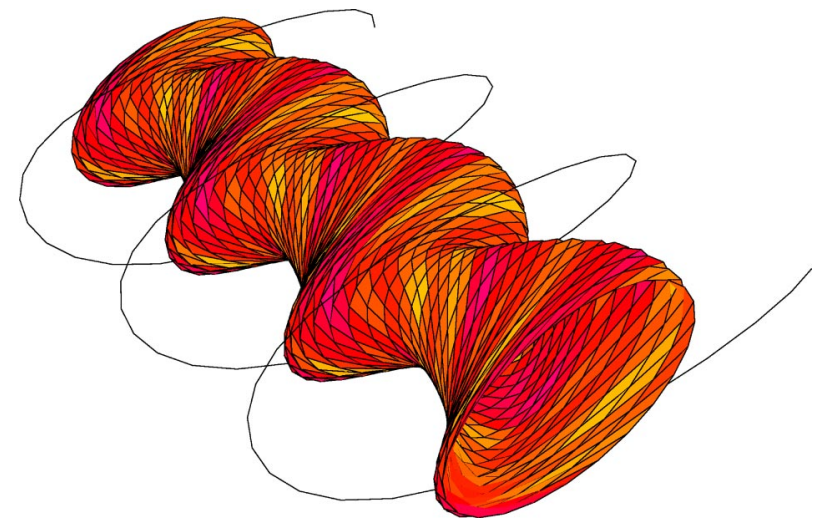

FIG. 3. Sketch of a core with ripple in the form of a snake wave.

second term is the constant radius of the core in case of absence of a wave, and the third term represents the wave shape. It can be seen from Eq. (16) that for snake waves a net hydrodynamic force is possible in $\epsilon^{2}$ approximation. So even when the wave shape in the $x$ direction for the snake wave is chosen to be equal to the one for the bamboo wave, the $\theta$ dependence of the snake wave changes the calculation profoundly.

We have studied core-annular flow for a core with a snake wave in more quantitative detail. The dependence on $\theta$ is clear from Eq. (21). The shape in the $x$ direction was chosen in such a way that at $\theta=0$ it was identical to the shape of a bamboo wave. This was done according to the following procedure. We selected $\theta=0$. In that case in Eq. (21) only $\cos n \pi x / l$ and $\sin n \pi x / l$ terms are present with coefficients $\left(a_{o, n}+a_{1, n}\right)$ and $\left(b_{o, n}+b_{1, n}\right)$. These coefficients were determined by selecting a bamboo-wave shape from Bai et al. [5] and calculating the Fourier components for this wave. The contribution of the Fourier components was then split over $a_{o, n}$ and $a_{1, n}$ and over $b_{o, n}$ and $b_{1, n}$ in such a way that the snake wave shown in Fig. 3 was found. As with the $\theta$ dependence many more possibilities exist for the $x$ dependence.

In our calculation for the core with a snake wave we studied the flow of the liquid in the annular layer. A helicallike flow field was found. Some typical streamlines are shown in Fig. 3. This flow field is different from the one calculated by Ooms and Beckers [11] for the case of a sawtooth-shape wave. In that case an oscillatory-like flow field was found: superposed on the main flow in the length direction of the pipe there were oscillating secondary flows on both sides of the core.

For the snake wave with $a_{m, n}$ and $b_{m, n}$ coefficients as described above we have calculated the dependence of $\Delta \rho / \rho$ as a function of the relevant parameters given by Eq. (17). For that purpose the eccentricity parameter $a_{1,0}$ and the amplitude parameter $\epsilon$ were chosen to have certain values. Some results are given in Fig. 4 and Fig. 5. In Fig. 4 the (dimensionless) density difference (which can be counterbalanced) is shown as function of the (dimensionless) thickness of the annular layer for three values of the ratio Fr/Re. In Fig. 5 it is given as a function of the (dimensionless) wavelength. It is clear that the film thickness has a significant influence, whereas the dependence on the wavelength is negligible. The 


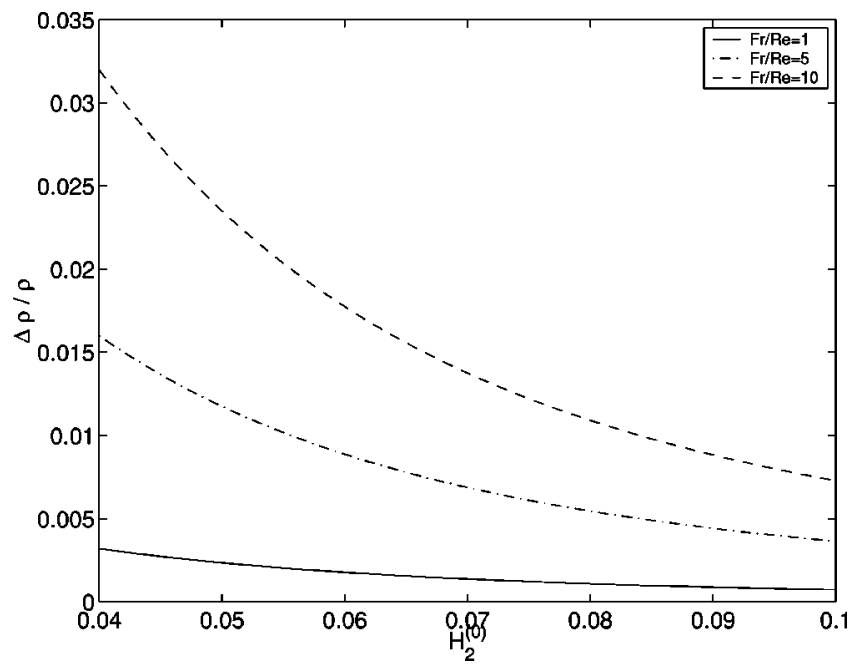

FIG. 4. $\Delta \rho / \rho$ as function of $H_{2}^{(0)}$ for three values of $\mathrm{Fr} / \mathrm{Re}$ $\left(a_{1,0}=0.5, \epsilon=0.02\right)$.

dependence on $\mathrm{Fr} / \mathrm{Re}=W \nu / g R^{2}$ is also considerable. $\nu$ is the kinematic viscosity of the liquid. With increasing values of $\mathrm{Fr} / \mathrm{Re}$ larger density differences can be counterbalanced.

We have made some additional calculations for the case of oil-water core-annular flow. We choose $R=0.1 \mathrm{~m}, l$ $=0.01 \mathrm{~m}, \mu=0.001 \mathrm{~Pa} \mathrm{~s}, \epsilon=0.01$, and $a_{1,0}=0.5$. For these value of the parameters we show in Fig. 6 the levitation force on the core as a function of the core velocity. Also three lines are given, which indicate which levitation force is needed to counterbalance density differences of $\Delta \rho=1.1 \mathrm{~kg} / \mathrm{m}^{3}, \Delta \rho$ $=5 \mathrm{~kg} / \mathrm{m}^{3}$, and $\Delta \rho=10 \mathrm{~kg} / \mathrm{m}^{3}$. In practice density differences between oil and water are of the order $10 \mathrm{~kg} / \mathrm{m}^{3}$. As can be seen oil velocities of the order $0.5 \mathrm{~m} / \mathrm{s}$ are sufficient to levitate the oil core. Such velocities are certainly possible in practice. Similar results for oil-water core-annular flow are given in Fig. 7 and Fig. 8. They show the dependence of the levitation force on the wavelength and on the water film thickness, respectively. The levitation force increases with increasing wavelength and decreasing water film thickness.

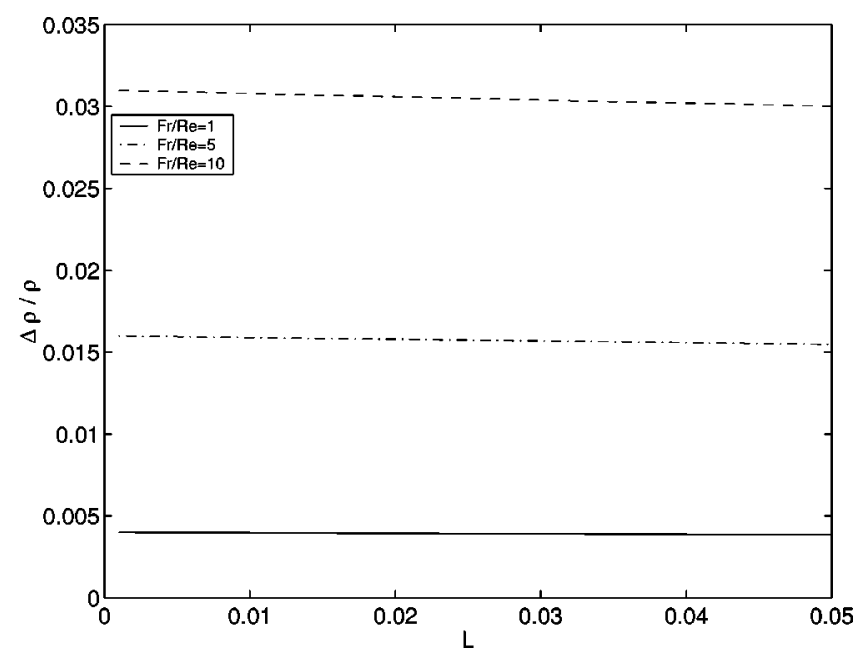

FIG. 5. $\Delta \rho / \rho$ as function of $L$ for three values of $\mathrm{Fr} / \mathrm{Re}\left(a_{1,0}\right.$ $=0.5, \epsilon=0.02$ ).

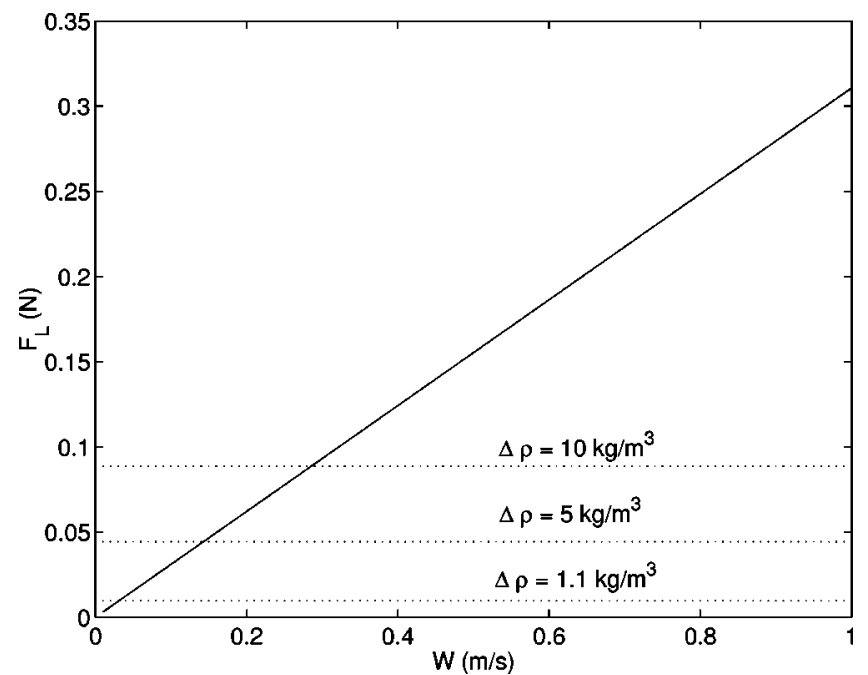

FIG. 6. Levitation force as function of velocity $W\left(a_{1,0}=0.5\right.$, $\left.R=0.1 \mathrm{~m}, l=0.03 \mathrm{~m}, \mu=0.001 \mathrm{Pas}, \epsilon=0.01, h_{2}^{(0)}=0.002 \mathrm{~m}\right)$.

Finally we show in Fig. 9 the axial force on the core as function of the axial velocity $W$. The contributions of the main flow and of the secondary flow are plotted separately. At small core velocities the contribution of the secondary flow is dominant. At large core velocities the contribution of the secondary flow becomes negligible and the frictional force is mainly due to the flow in the axial direction of the pipe.

\section{DISCUSSION}

A theoretical model, based on the hydrodynamiclubrication theory, for core-annular flow through a pipe has been developed. According to this model the movement of the rippled core with respect to the pipe wall induces pressure variations and secondary flows in the annular liquid, which can exert a net lubrication force on the core in the

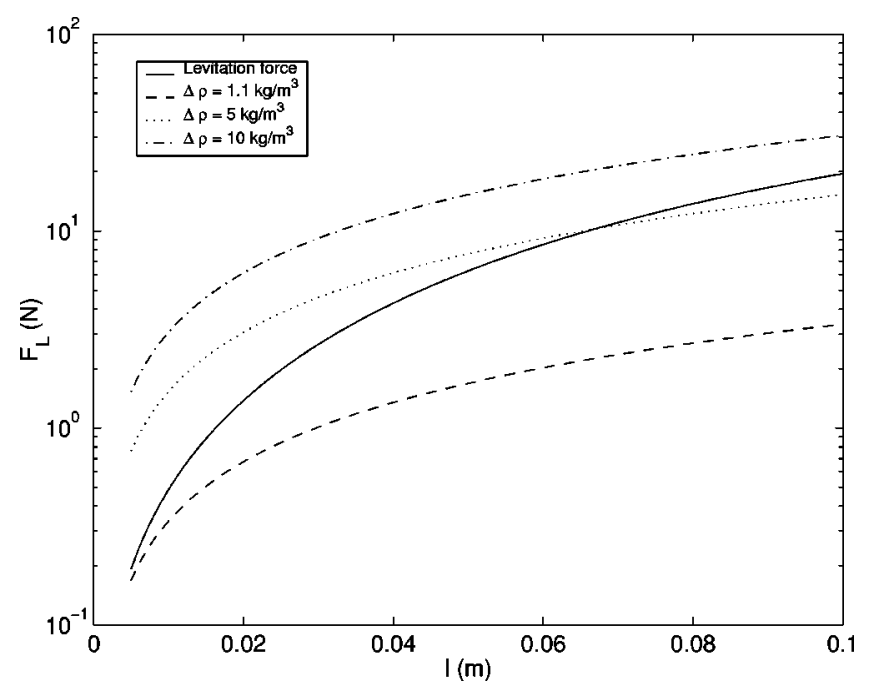

FIG. 7. Levitation force as function of wavelength $l\left(a_{1,0}\right.$ $=0.5, \quad R=0.1 \mathrm{~m}, \quad h_{2}^{(0)}=0.01 \mathrm{~m}, \quad \mu=0.001 \mathrm{Pas}, \quad \epsilon=0.01, \quad W$ $=1 \mathrm{~m} / \mathrm{s}$ ). 


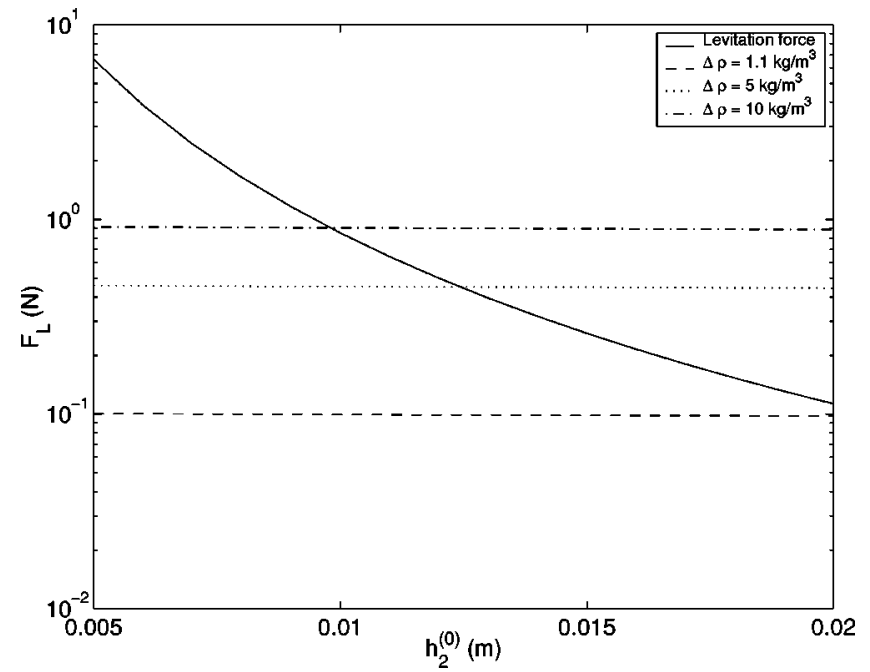

FIG. 8. Levitation force as function of water film thickness $h_{2}^{(0)}$ $\left(a_{1,0}=0.5, \quad R=0.1 \mathrm{~m}, \quad l=0.03 \mathrm{~m}, \quad \mu=0.001 \mathrm{Pas}, \quad \epsilon=0.01, \quad W\right.$ $=1 \mathrm{~m} / \mathrm{s})$.

vertical direction. For a ripple in the form of a snake wave this force can be so large that it counterbalances the buoyancy force on the core allowing a steady core-annular flow to arise. For a ripple in the form of a bamboo wave this is not possible.

In our calculations we have neglected inertial forces. As was shown by Bai et al. [5] inertial forces are likely responsible for the levitation of a core with bamboo waves. Dependent on the Reynolds number of the liquid flow in the annulus it can, therefore, be expected that also for snake waves the inertial forces can have a significant influence on core levitation. For that reason we will in our future work include the inertial forces when studying core-annular flow with

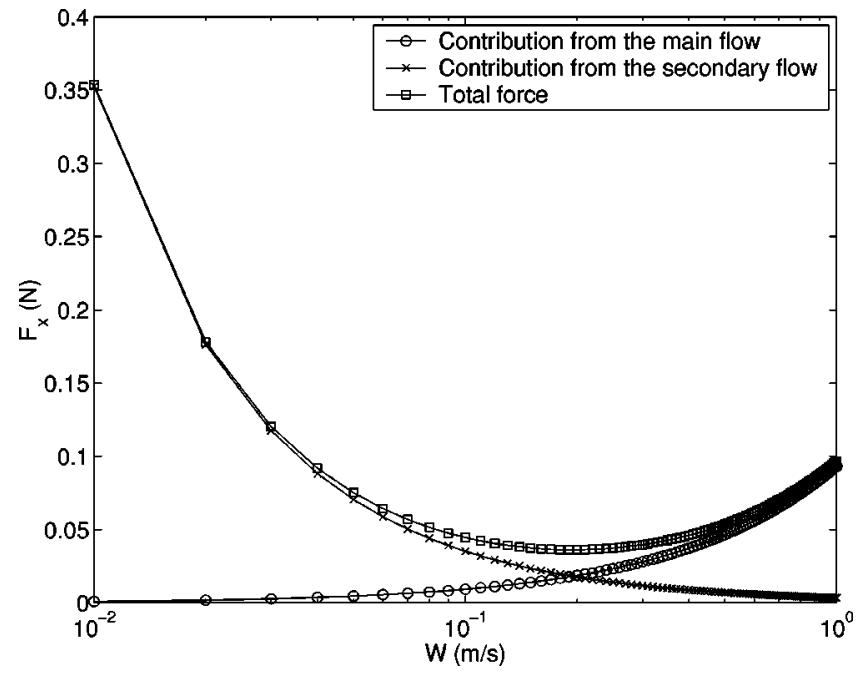

FIG. 9. Axial force as function of the oil core velocity $\left(a_{1,0}\right.$ $=0.5, \quad R=0.1 \mathrm{~m}, \quad l=0.03 \mathrm{~m}, \quad \mu=0.001 \mathrm{~Pa} \mathrm{~s}, \quad \epsilon=0.01, \quad h_{2}^{(0)}$ $=0.002 \mathrm{~m})$.

snake waves at the core surface. Also the influence of the turbulence in the annular layer will be included following the work of Oliemans [15].

At the moment three theoretical models exist for explaining the counterbalancing of the buoyancy force: (1) the hydrodynamic-lubrication-force model of Ooms, Oliemans, and co-workers, (2) the inertial-pressure-force model of Bai, Joseph, and co-workers, and (3) the interfacial-tension-force model of Bannwarth. It is necessary to compare the relative importance of the levitation mechanisms described by these models as function of the values of the relevant parameters of core-annular flow. We will, therefore, pay attention to this comparison.
[1] D.D. Joseph and Y.Y. Renardy, Fundamentals of Two-Fluid Dynamics, Part II: Lubricated Transport, Drops and Miscible Liquids (Springel-Verlag, New York, 1993).

[2] R.V.A. Oliemans and G. Ooms, in Core-annular Flow of Oil and Water Through a Pipeline, edited by G.F. Hewitt, J.M. Delhaye, and N. Zuber, Multiphase Science and Technology Vol. 2 (Hemisphere, Washington, D.C., 1986).

[3] D.D. Joseph, R. Bai, K.P. Chen, and Y.Y. Renardy, Annu. Rev. Fluid Mech. 29, 65 (1999).

[4] G. Ooms, Appl. Sci. Res. 26, 147 (1972).

[5] R. Bai, K. Kelkar, and D.D. Joseph, J. Fluid Mech. 327, 1 (1996).

[6] R. Bai, K. Chen, and D.D. Joseph, J. Fluid Mech. 240, 97
(1992).

[7] Y.Y. Renardy, J. Fluid Mech. 340, 297 (1997).

[8] J. Li and Y.Y. Renardi, J. Fluid Mech. 391, 123 (1999).

[9] C. Kouris and J. Tsamopoulos, Phys. Fluids 13, 841 (2001).

[10] T. Ko, H.G. Choi, R. Bai, and D.D. Joseph, Int. J. Multiphase Flow 28, 1205 (2002).

[11] G. Ooms and H.L. Beckers, Appl. Sci. Res. 26, 321 (1972).

[12] G. Ooms, A. Segal, A.J. van der Wees, R. Meerhoff, and R.V.A. Oliemans, Int. J. Multiphase Flow 10, 41 (1984).

[13] A.C. Bannwart, J. Energy Resour. Technol. 123, 127 (2001).

[14] R. Kronig (private communication).

[15] R.V.A. Oliemans, Ph.D. thesis, Delft University of Technology, 1986. 